\title{
A generalization for surfaces using a line of curvature in Lie group
}

\author{
Dae Won Yoon ${ }^{1}$ (D), Zühal Küçükarslan Yüzbaş ${ }^{* 2}$ (D) \\ ${ }^{1}$ Department of Mathematics Education and RINS, Gyeongsang National University, Jinju 52828, \\ Republic of Korea \\ ${ }^{2}$ Department of Mathematics, Faculty of Science, Frat University, 23119 Elazig, Turkey
}

\begin{abstract}
In this study, we investigate how to construct surfaces using a line of curvature in a 3dimensional Lie group. Then, by utilizing the Frenet frame, we give the conditions that a curve becomes a line of curvature on a surface when the marching-scale functions are more general expressions. After then, we provide some crucial examples of how efficient our method is on these surfaces.
\end{abstract}

Mathematics Subject Classification (2010). 53A05, 22E15

Keywords. surface family, Lie Group, line of curvature

\section{Introduction}

A line of curvature is one of the important characteristic curves on a surface and has been a long standing research focus in differential geometry. A line of curvature on a surface is a curve on a surface whose tangent at each point is the principal direction of the surface.

Traditionally, most of the studies for a line of curvature deal with finding and computing them. Also, the researches on the line of curvature are determined by three forms as the representations of the surface, that is, parametric form, implicit form and discrete sampling form [8]. In [6,8] Li et al. studied the method of how to establish parametric surfaces using a line of curvature. Also, Ergun et al. [5] gave a parametric surface family using a spacelike (timelike) line of curvature in Minkowski space. In [10], the authors described a method to extract the generic features of free form parametric surfaces for shape interrogation. Che and Paul [1] studied an approach to analyze and compute the lines of curvature and their differential geometry defined on implicit surfaces. Also, in [13] authors presented a scheme for computing and visualizing the lines of curvature defined on the implicit surface. On the other hand, many geometers considered recently the reverse problems of the traditional research mentioned above. The basic idea of the reverse problems is to regard the wanted surfaces as an extension from the given line of curvature, and represented it in terms of some functions and the Frenet frame of a spatial curve. Li et al. [6] introduced firstly the method of how to construct surfaces passing through a line of curvature and gave interesting examples in Euclidean space. In [7], the authors proposed a new method to

\footnotetext{
*Corresponding Author.

Email addresses: dwyoon@gnu.ac.kr (D.W. Yoon), zuhal2387@yahoo.com.tr (Z. Küçükarslan Yüzbaşı)

Received: 25.12.2019; Accepted: 01.08.2020
} 
construct a developable surface with a given curve as the line of curvature of it. After, in $[5,9]$ authors gave the necessary and sufficient conditions for the given non-null curve to be the line of curvature on a spacelike or timelike surface in Minkowski space and investigated the problem of constructing a surface from a given non-null line of curvature.

In this study, we improve the method of $\mathrm{Li}$ et al. $[6,8]$ to get parametric surfaces using a line of curvature in a Lie group. We also consider marching-scale functions as more general expressions and derive the conditions that the given curve becomes a common line of curvature on the surface. We furthermore investigate some representative curves that are given to show the parametric surfaces possessing these curves as lines of curvature.

\section{Preliminaries}

Let $G$ be a Lie group with a bi-invariant metric $\langle$,$\rangle and \mathfrak{g}$ denote the Lie algebra of $G$. Denote by $D$ the corresponding Levi-Civita connection of $G$. Then $\mathfrak{g}$ is isomorphic to $T_{e} G$, where $e$ is the identity (the neutral element) of $G$. For the bi-invariant metric $\langle$,$\rangle on G$, thus we get

$$
\langle X,[Y, Z]\rangle=\langle[X, Y], Z\rangle
$$

and

$$
D_{X} Y=\frac{1}{2}[X, Y]
$$

for all $X, Y, Z \in \mathfrak{g}$. Let $r: I \subset \mathbb{R} \rightarrow G$ be a curve parametrized by the arc-length $s$ and $\left\{X_{1}, X_{2}, \ldots, X_{n}\right\}$ be an orthonormal basis of $\mathfrak{g}$. We can express any two vector fields $V$ and $W$ along $r$ as $V=\sum_{i=1}^{n} v_{i} X_{i}$ and $W=\sum_{i=1}^{n} w_{i} X_{i}$, where $v_{i}: I \rightarrow \mathbb{R}$ and $w_{i}: I \rightarrow \mathbb{R}$ are smooth functions. Also, the Lie bracket of $V$ and $W$ is written by

$$
[V, W]=\sum_{i, j=1}^{n} v_{i} w_{j}\left[X_{i}, X_{j}\right]
$$

$T=r^{\prime}$, where $r^{\prime}=\frac{d r}{d s}$, and $W^{\prime}=\sum_{i=1}^{n} w_{i}^{\prime} X_{i}$, where $w_{i}^{\prime}=\frac{d w_{i}}{d s}$. Then we get

$$
D_{r^{\prime}} W=W^{\prime}+\frac{1}{2}[T, W] \text {. }
$$

Let $\alpha: I \subset \mathbb{R} \rightarrow G$ be a curve in a 3 -dimensional Lie group $G$ with the Frenet frame $\{T, N, B\}$. Then the Frenet formulas are written by

$$
D_{T} T=k_{1} N, D_{T} N=-k_{1} T+k_{2} B, D_{T} B=-k_{2} N,
$$

where $T=\alpha^{\prime}, k_{1}=\left\|D_{T} T\right\|=\left\|T^{\prime}\right\|$ and $k_{2}$ are the curvatures of $\alpha$ in $G$.

Also, a smooth function $\overline{k_{2}}(s)$ is defined by $[2,12]$ :

$$
\overline{k_{2}}=\frac{1}{2}\langle T,[N, B]\rangle \text {. }
$$

Proposition 2.1. (cf. [12]) Let $\alpha: I \subset \mathbb{R} \rightarrow G$ be a curve parametrized by the arc-length $s$ in a 3-dimensional Lie group $G$. Then, the following relations hold:

$$
\begin{aligned}
& {[T, N]=\langle[T, N], B\rangle B=2 \overline{k_{2}} B,} \\
& {[B, T]=\langle[B, T], N\rangle N=2 \overline{k_{2}} N,} \\
& {[N, B]=\langle[N, B], T\rangle T=2 \overline{k_{2}} T .}
\end{aligned}
$$

Remark 2.2. Let $G$ be a 3 -dimensional Lie group with a bi-invariant metric. Then it is one of the Lie groups $S O(3), S^{3}$ or a commutative group and the following statements hold $([2,4])$ :

(i) If $G$ is $S O(3)$, then $\bar{k}_{2}(s)=\frac{1}{2}$.

(ii) If $G$ is $S^{3} \cong S U(2)$, then $\bar{k}_{2}(s)=1$. 
(iii) If $G$ is a commutative group, then $\bar{k}_{2}(s)=0$.

The normal vector $\eta$ of the surface $\Psi(s, t)$ is given by

$$
\eta(s, t)=\frac{\partial \Psi(s, t)}{\partial s} \times \frac{\partial \Psi(s, t)}{\partial t} .
$$

\section{General surface family with a line of curvature}

In this section, we construct a surface family with a line of curvature in a 3-dimensional Lie group $G$. We give the conditions for the given curve to be the line of curvature for the parametric surface when the marching-scale functions are given by the three types of expressions.

Let $\alpha(s)$ be a curve as defined on the Frenet frame $\{T, N, B\}$. Then we can give the parametric form of a surface given by the curve $\alpha(s)$ as follows:

$$
\begin{gathered}
\Psi(s, t)=\alpha(s)+x(s, t) T(s)+y(s, t) N(s)+z(s, t) B(s), \\
L_{1} \leq s \leq L_{2} \text { and } T_{1} \leq t \leq T_{2},
\end{gathered}
$$

where $x(s, t), y(s, t)$ and $z(s, t)$ are all $C^{1}$ functions. These functions are said to be the marching-scale functions.

A curve $\alpha(s)$ on the surface $\Psi(s, t)$ in $G$ is said to be isoparametric if the equations

$$
\begin{gathered}
x\left(s, t_{0}\right)=y\left(s, t_{0}\right)=z\left(s, t_{0}\right)=0, \\
L_{1} \leq s \leq L_{2} \text { and } t_{0} \in\left[T_{1}, T_{2}\right],
\end{gathered}
$$

hold, where $t_{0}$ is a parameter such that $\alpha(s)=\Psi\left(s, t_{0}\right)$.

The next theorem is well known and useful:

Theorem 3.1. ([11]) A curve on a surface is a line of curvature if and only if the surface normals along the curve form a developable surface.

Now, we show the conditions for $\alpha(s)$ being the line of curvature on $\Psi(s, t)$.

Let the normal surface of $\alpha(s)$ be

$$
\Phi(s, t)=\alpha(s)+t \eta_{1},
$$

where $\eta_{1}=\cos \vartheta N+\sin \vartheta B$ is a vector orthogonal to the curve $\alpha(s)$ and $N, B$ are the principal normal and the binormal of the curve $\alpha(s)$, respectively.

It is well known that the normal surface $\Phi(s, t)$ is developable if and only if $\operatorname{det}\left(\alpha^{\prime}, \eta_{1}, \eta_{1}^{\prime}\right)=$ 0 [3]. Thus we obtain

$$
\begin{aligned}
\operatorname{det}\left(\alpha^{\prime}, \eta_{1}, \eta_{1}^{\prime}\right)=0 & \\
& \Longleftrightarrow\left(\begin{array}{c}
T, \cos \vartheta N+\sin \vartheta B,-k_{1} \cos \vartheta T-\left(\vartheta^{\prime} \sin \vartheta+\sin \vartheta\left(k_{2}-\overline{k_{2}}\right)\right) N \\
+\left(\vartheta^{\prime} \cos \vartheta+\cos \vartheta\left(k_{2}-\overline{k_{2}}\right)\right) B
\end{array}\right)=0 \\
& \Longleftrightarrow \vartheta^{\prime}+\left(k_{2}-\overline{k_{2}}\right)=0 \\
& \Longleftrightarrow \vartheta^{\prime}=-\left(k_{2}-\overline{k_{2}}\right) \\
& \Longleftrightarrow \vartheta=-\int_{s_{0}}^{s}\left(k_{2}-\overline{k_{2}}\right) d s+\vartheta_{0},
\end{aligned}
$$

where $s_{0}$ is the initial value of $s$ and $\vartheta_{0}=\vartheta\left(s_{0}\right)$. Along this study, we suppose that $s_{0}=0$. 
To compute the normal vector of $\Psi(s, t)$, we obtain the followings:

$$
\begin{aligned}
\frac{\partial \Psi(s, t)}{\partial s}= & \left(1+\frac{\partial x(s, t)}{\partial s}-y(s, t) k_{1}(s)\right) T(s) \\
& +\left(x(s, t) k_{1}(s)+\frac{\partial y(s, t)}{\partial s}-z(s, t)\left(k_{2}(s)-\overline{k_{2}}(s)\right)\right) N(s) \\
& +\left(\frac{\partial z(s, t)}{\partial s}+y(s, t)\left(k_{2}(s)-\overline{k_{2}}(s)\right)\right) B(s), \\
\frac{\partial \Psi(s, t)}{\partial t}= & \frac{\partial x(s, t)}{\partial t} T(s)+\frac{\partial y(s, t)}{\partial t} N(s)+\frac{\partial z(s, t)}{\partial t} B(s),
\end{aligned}
$$

which implies the normal vector $\eta$ of the surface $\Psi(s, t)$ is given by

$$
\begin{aligned}
\eta(s, t)= & \frac{\partial \Psi(s, t)}{\partial s} \times \frac{\partial \Psi(s, t)}{\partial t} \\
= & {\left[\left(x(s, t) k_{1}(s)+\frac{\partial y(s, t)}{\partial s}-z(s, t)\left(k_{2}(s)-\overline{k_{2}}(s)\right)\right) \frac{\partial z(s, t)}{\partial t}\right.} \\
& \left.-\left(\frac{\partial z(s, t)}{\partial s}+y(s, t)\left(k_{2}(s)-\overline{k_{2}}(s)\right)\right) \frac{\partial y(s, t)}{\partial t}\right] T(s) \\
& +\left[-\left(1+\frac{\partial x(s, t)}{\partial s}-y(s, t) k_{1}(s)\right) \frac{\partial z(s, t)}{\partial t}\right. \\
& \left.+\left(\frac{\partial z(s, t)}{\partial s}+y(s, t)\left(k_{2}(s)-\overline{k_{2}}(s)\right)\right) \frac{\partial x(s, t)}{\partial t}\right] N(s) \\
& +\left[\left(1+\frac{\partial x(s, t)}{\partial s}-y(s, t) k_{1}(s)\right) \frac{\partial y(s, t)}{\partial t}\right. \\
& \left.-\left(x(s, t) k_{1}(s)+\frac{\partial y(s, t)}{\partial s}-z(s, t)\left(k_{2}(s)-\overline{k_{2}}(s)\right)\right) \frac{\partial x(s, t)}{\partial t}\right] B(s) .
\end{aligned}
$$

It follows that we have

$$
\eta\left(s, t_{0}\right)=\psi_{1}\left(s, t_{0}\right) T(s)+\psi_{2}\left(s, t_{0}\right) N(s)+\psi_{3}\left(s, t_{0}\right) B(s),
$$

where

$$
\begin{array}{r}
\psi_{1}\left(s, t_{0}\right)=\left.\left.\frac{\partial y(s, t)}{\partial s}\right|_{\left(s, t_{0}\right)} \frac{\partial z(s, t)}{\partial t}\right|_{\left(s, t_{0}\right)}-\left.\left.\frac{\partial z(s, t)}{\partial s}\right|_{\left(s, t_{0}\right)} \frac{\partial y(s, t)}{\partial t}\right|_{\left(s, t_{0}\right)}, \\
\psi_{2}\left(s, t_{0}\right)=-\left.\left(1+\left.\frac{\partial x(s, t)}{\partial s}\right|_{\left(s, t_{0}\right)}\right) \frac{\partial z(s, t)}{\partial t}\right|_{\left(s, t_{0}\right)}-\left.\left.\frac{\partial z(s, t)}{\partial s}\right|_{\left(s, t_{0}\right)} \frac{\partial x(s, t)}{\partial t}\right|_{\left(s, t_{0}\right)} \\
\psi_{3}\left(s, t_{0}\right)=\left.\left(1+\left.\frac{\partial x(s, t)}{\partial s}\right|_{\left(s, t_{0}\right)}\right) \frac{\partial y(s, t)}{\partial t}\right|_{\left(s, t_{0}\right)}-\left.\left.\frac{\partial y(s, t)}{\partial s}\right|_{\left(s, t_{0}\right)} \frac{\partial x(s, t)}{\partial t}\right|_{\left(s, t_{0}\right)} .
\end{array}
$$

On the other hand, a curve $\alpha(s)$ is a line of curvature on the surface $\Psi(s, t)$ if and only if the normal vector $\eta_{1}(s)$ of the curve is parallel to the normal vector $\eta\left(s, t_{0}\right)$ of the surface, that is, $\eta_{1}(s) \| \eta\left(s, t_{0}\right)$. From this fact, there exists a function $\lambda(s) \neq 0$ such that

$$
\psi_{1}\left(s, t_{0}\right)=0, \psi_{2}\left(s, t_{0}\right)=\lambda(s) \cos \vartheta \text { and } \psi_{3}\left(s, t_{0}\right)=\lambda(s) \sin \vartheta .
$$

Thus, we have the following theorem. 
Theorem 3.2. A given curve $\alpha: I \subset \mathbb{R} \rightarrow G$ is a line of curvature on the surface $\Psi(s, t)$ with the parametrization (3.1) in a 3-dimensional Lie group $G$ if and only if

$$
\begin{aligned}
x\left(s, t_{0}\right) & =y\left(s, t_{0}\right)=z\left(s, t_{0}\right)=0, \\
\psi_{1}\left(s, t_{0}\right) & =0, \psi_{2}\left(s, t_{0}\right)=\lambda(s) \cos \vartheta \text { and } \psi_{3}\left(s, t_{0}\right)=\lambda(s) \sin \vartheta, \\
\vartheta(s) & =-\int_{s_{0}}^{s}\left(k_{2}-\overline{k_{2}}\right) d s+\vartheta_{0},
\end{aligned}
$$

where $s \in\left[L_{1}, L_{2}\right], t_{0} \in\left[T_{1}, T_{2}\right]$ and the functions $\vartheta(s)$ and $\lambda(s) \neq 0$ are called controlling functions.

Therefore, we call the set of surfaces satisfying Theorem 3.2 a surface family with a common line of curvature in $G$.

Remark 3.3. Generally we can take $\vartheta_{0}=0$. According to $x\left(s, t_{0}\right)=y\left(s, t_{0}\right)=z\left(s, t_{0}\right)=$ 0 , we have $\left.\frac{\partial x(s, t)}{\partial s}\right|_{\left(s, t_{0}\right)}=\left.\frac{\partial y(s, t)}{\partial s}\right|_{\left(s, t_{0}\right)}=\left.\frac{\partial z(s, t)}{\partial s}\right|_{\left(s, t_{0}\right)}=0$.

Then from the above conditions and using (3.3), we have

$$
\begin{aligned}
& \psi_{2}\left(s, t_{0}\right)=-\left.\frac{\partial z(s, t)}{\partial t}\right|_{\left(s, t_{0}\right)}, \\
& \psi_{3}\left(s, t_{0}\right)=\left.\frac{\partial y(s, t)}{\partial t}\right|_{\left(s, t_{0}\right)} .
\end{aligned}
$$

Thus (3.4) can be rewritten simply

$$
\begin{aligned}
x\left(s, t_{0}\right) & =y\left(s, t_{0}\right)=z\left(s, t_{0}\right)=0, \\
\left.\frac{\partial y(s, t)}{\partial t}\right|_{\left(s, t_{0}\right)} & =\lambda(s) \sin \vartheta \\
\left.\frac{\partial z(s, t)}{\partial t}\right|_{\left(s, t_{0}\right)} & =-\lambda(s) \cos \vartheta .
\end{aligned}
$$

For simplification and analysis, we note that there are three types of the marching-scale functions $x(s, t), y(s, t)$ and $z(s, t)$ as follows:

1) Considering

$$
\begin{aligned}
& x(s, t)=p(s) X(t), \\
& y(s, t)=q(s) Y(t), \\
& z(s, t)=r(s) Z(t),
\end{aligned}
$$

where $p(s), q(s), r(s), X(t), Y(t)$ and $Z(t)$ are $C^{1}$ functions, and $p(s), q(s)$ and $r(s)$ are not identically zero.

2) Considering

$$
\begin{aligned}
& x(s, t)=\sum_{i=1}^{k} a_{1 i} p(s)^{i} X(t)^{i}, \\
& y(s, t)=\sum_{i=1}^{k} a_{2 i} q(s)^{i} Y(t)^{i}, \\
& z(s, t)=\sum_{i=1}^{k} a_{3 i} r(s)^{i} Z(t)^{i},
\end{aligned}
$$

then 


$$
\begin{aligned}
& \left.\frac{\partial y(s, t)}{\partial t}\right|_{\left(s, t_{0}\right)}=\sum_{i=1}^{k} k a_{2 i} q(s)^{k} Y\left(t_{0}\right)^{k-1} Y^{\prime}\left(t_{0}\right), \\
& \left.\frac{\partial z(s, t)}{\partial t}\right|_{\left(s, t_{0}\right)}=\sum_{i=1}^{k} k a_{3 i} r(s)^{k} Z\left(t_{0}\right)^{k-1} Z^{\prime}\left(t_{0}\right) .
\end{aligned}
$$

3) Considering

$$
\begin{aligned}
& x(s, t)=f\left(\sum_{i=1}^{k} a_{1 i} p(s)^{i} X(t)^{i}\right), \\
& y(s, t)=g\left(\sum_{i=1}^{k} a_{2 i} q(s)^{i} Y(t)^{i}\right), \\
& z(s, t)=h\left(\sum_{i=1}^{k} a_{3 i} r(s)^{i} Z(t)^{i}\right),
\end{aligned}
$$

where $a_{i j} \in R(i=1,2,3 ; j=1,2, \ldots, k)$, and $X(t), Y(t), Z(t), f, g, h$ are smooth functions.

Corollary 3.4. According to the condition (3.5) and by simple computation, the conditions of the curve $\alpha: I \subset \mathbb{R} \rightarrow G$ with a line of curvature on the surface $\Psi(s, t)$ in terms of three types of the marching-scale functions are given by, such that $X\left(t_{0}\right)=Y\left(t_{0}\right)=Z\left(t_{0}\right)=0$, respectively,

1)

$$
\begin{aligned}
& q(s) Y^{\prime}\left(t_{0}\right)=\lambda(s) \sin \vartheta, \\
& r(s) Z^{\prime}\left(t_{0}\right)=-\lambda(s) \cos \vartheta .
\end{aligned}
$$

2) From the equations (3.8),

$$
\begin{aligned}
& a_{21} q(s) Y^{\prime}\left(t_{0}\right)=\lambda(s) \sin \vartheta, \\
& a_{31} r(s) Z^{\prime}\left(t_{0}\right)=-\lambda(s) \cos \vartheta .
\end{aligned}
$$

3)

$$
\begin{aligned}
& f(0)=g(0)=h(0)=0, \\
& g^{\prime}(0) a_{21} q(s) Y^{\prime}\left(t_{0}\right)=\lambda(s) \sin \vartheta, \\
& h^{\prime}(0) a_{31} r(s) Z^{\prime}\left(t_{0}\right)=-\lambda(s) \cos \vartheta .
\end{aligned}
$$

We analyze the conditions (3.10), (3.11) and (3.12) according to the different expressions of $\vartheta(s)$ as follows:

Case 1. If $\alpha: I \subset \mathbb{R} \rightarrow G$ is a curve with $\left(k_{2}-\overline{k_{2}}\right)(s) \neq 0$, then $\vartheta(s)$ is a nonconstant function of a variable $s$, for $X\left(t_{0}\right)=Y\left(t_{0}\right)=Z\left(t_{0}\right)=0$, the conditions (3.10), (3.11) and (3.12) can be given as, respectively,

1)

$$
\begin{aligned}
Y^{\prime}\left(t_{0}\right) & =\frac{\lambda(s)}{q(s)} \sin \vartheta, \\
Z^{\prime}\left(t_{0}\right) & =-\frac{\lambda(s)}{r(s)} \cos \vartheta .
\end{aligned}
$$


2)

$$
\begin{aligned}
Y^{\prime}\left(t_{0}\right) & =\frac{\lambda(s)}{a_{21} q(s)} \sin \vartheta=\nu_{1}, \\
Z^{\prime}\left(t_{0}\right) & =-\frac{\lambda(s)}{a_{31} r(s)} \cos \vartheta=-\nu_{2} .
\end{aligned}
$$

In this case we can take $\lambda(s)=\nu_{1} a_{21} \lambda_{1}(s), q(s)=\lambda_{1}(s) \sin \vartheta$ and $r(s)=\frac{\nu_{1} a_{21} \lambda_{1}(s)}{\nu_{2} a_{31}} \cos \vartheta$. 3)

$$
\begin{aligned}
Y^{\prime}\left(t_{0}\right) & =\frac{\lambda(s)}{g^{\prime}(0) a_{21} q(s)} \sin \vartheta=\nu_{1}, \\
Z^{\prime}\left(t_{0}\right) & =-\frac{\lambda(s)}{h^{\prime}(0) a_{31} r(s)} \cos \vartheta=-\nu_{2} .
\end{aligned}
$$

In this case, we can take $\lambda(s)=\nu_{1} g^{\prime}(0) a_{21} \lambda_{1}(s), q(s)=\lambda_{1}(s) \sin \vartheta$ and $r(s)=\frac{\nu_{1} g^{\prime}(0) a_{21} \lambda_{1}(s)}{\nu_{2} h^{\prime}(0) a_{31}} \cos \vartheta$.

Case 2. This case is the same as all three types of marching-scale functions. If $\alpha: I \subset \mathbb{R} \rightarrow G$ is a curve with $\left(k_{2}-\overline{k_{2}}\right)(s)=0$, then $\vartheta(s)$ is constant. If $\vartheta=k \pi+\frac{\pi}{2}$, $k \in R$, i.e., $\sin \vartheta= \pm 1$ and $\cos \vartheta=0$, then in terms of the Frenet formula in $G$, we have binormal vector field $B$ of the curve $\alpha(s)$ is constant. Therefore $\eta_{1}=\cos \vartheta N+\sin \vartheta B$ $= \pm B$ is also a constant vector. In this case, the surface which passes through the given curve is the osculating plane of the curve. If $\vartheta \neq k \pi+\frac{\pi}{2}$, the condition is the same as Case 1.

Case 3. If $\left(k_{2}-\overline{k_{2}}\right)(s)=0$ and $\sin \vartheta=0$, then $\eta_{1}=\cos \vartheta N+\sin \vartheta B= \pm N$. Since the curve $\alpha(s)$ is a line of curvature, that is, $\eta_{1}(s) \| \eta\left(s, t_{0}\right)$, we get $\psi_{1}\left(s, t_{0}\right)=0, \psi_{2}\left(s, t_{0}\right) \neq 0$ and $\psi_{3}\left(s, t_{0}\right)=0$. Then the curve $\alpha(s)$ is not only a line of curvature but also a geodesic of the surface $\Psi(s, t)$. For $X\left(t_{0}\right)=Y\left(t_{0}\right)=Z\left(t_{0}\right)=0$, the conditions (3.10), (3.11) and (3.12) can be given as, respectively,

1)

$$
\begin{aligned}
& Y^{\prime}\left(t_{0}\right)=0, \\
& Z^{\prime}\left(t_{0}\right)= \pm \lambda(s) .
\end{aligned}
$$

2)

$$
\begin{aligned}
a_{21} & =0 \text { or } q(s)=0 \text { or } Y^{\prime}\left(t_{0}\right)=0, \\
Z^{\prime}\left(t_{0}\right) & = \pm \frac{\lambda(s)}{a_{31} r(s)}=\nu .
\end{aligned}
$$

In this case, we can take $\lambda(s)=\nu a_{31} \lambda_{1}(s)$ and $r(s)= \pm \lambda_{1}(s)$.

3)

$$
\begin{aligned}
f(0) & =g(0)=h(0)=0, \\
a_{21} & =0 \text { or } q(s)=0 \text { or } g^{\prime}(0)=0 \text { or } Y^{\prime}\left(t_{0}\right)=0, \\
Z^{\prime}\left(t_{0}\right) & = \pm \frac{\lambda(s)}{h^{\prime}(0) a_{31} r(s)}=\nu .
\end{aligned}
$$

In this case, we can take $\lambda(s)=\nu g^{\prime}(0) a_{21} \lambda_{1}(s)$ and $r(s)= \pm \lambda_{1}(s)$.

Example 3.5. Let the base curve $\alpha$ be

$$
\alpha(s)=(\cos s, \sin s, 0) .
$$




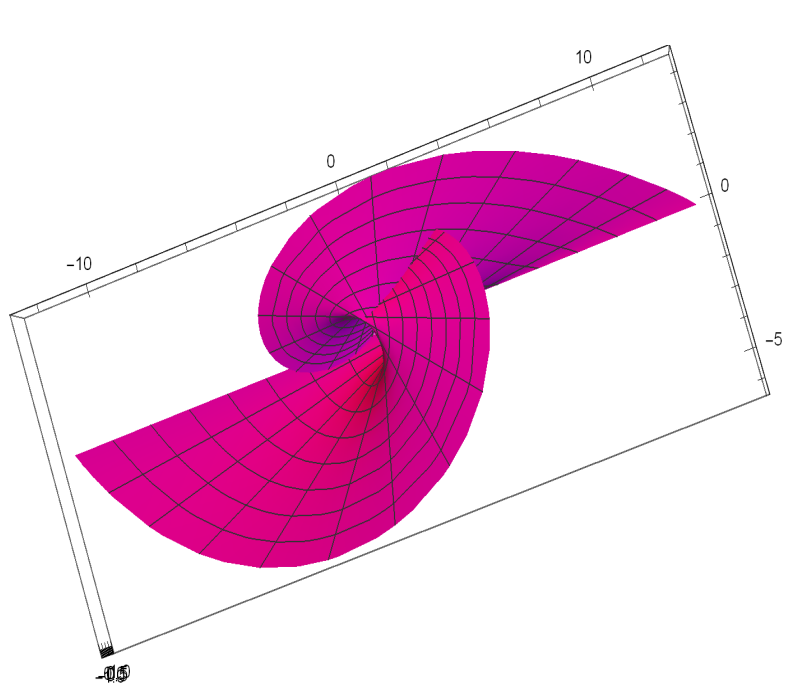

(a) $\Psi_{1}(s, t)$ with a line of curvature

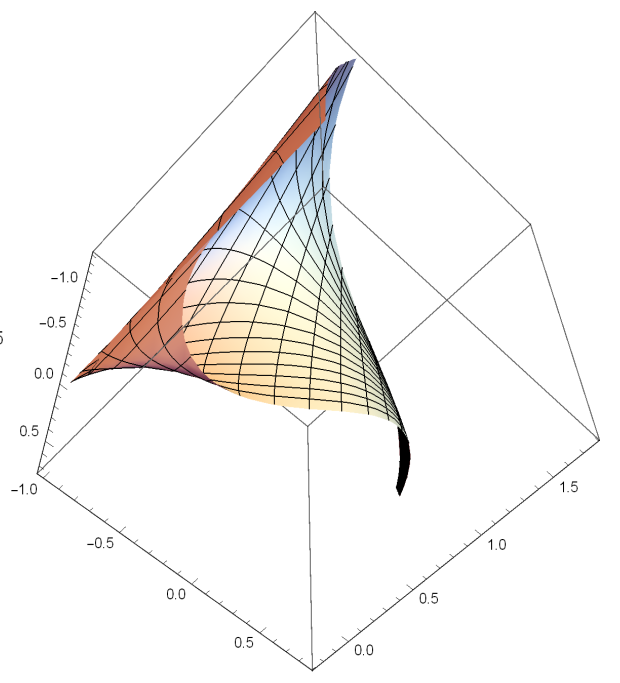

(b) $\Psi_{2}(s, t)$ with a line of curvature

Figure 1. A member of the surface family with marching-scale functions and its line of curvature.

Then we obtain

$$
\begin{aligned}
T(s) & =(-\sin s, \cos s, 0), \\
N(s) & =(-\cos s,-\sin s, 0), \\
B(s) & =(0,0,1),
\end{aligned}
$$

where $k_{1}=1, k_{2}=0, \overline{k_{2}}=\frac{1}{2}$ and $\vartheta(s)=\frac{s}{2}$.

a) If we consider $p(s)=q(s)=r(s)=1, \lambda(s)=s$ and

$$
\begin{aligned}
& x(s, t)=t s, \\
& y(s, t)=t s \sin \frac{s}{2}, \\
& z(s, t)=-t s \cos \frac{s}{2},
\end{aligned}
$$

at $t_{0}=0$ such that (3.13) is satisfied, then we get a member of this family in $G=S O(3)$ as drawn in Figure 1(a):

$$
\Psi_{1}(s, t)=\alpha(s)+t s T+t s \sin \frac{s}{2} N-t s \cos \frac{s}{2} B,
$$

where $0 \leq s \leq 2 \pi$ and $-1 \leq t \leq 1$.

b) Considering

$$
\begin{aligned}
& x(s, t)=0, \\
& y(s, t)=\sum_{i=1}^{2} a_{2 i}\left(\nu_{1} t \lambda_{1}(s) \sin \vartheta\right)^{i}, \\
& z(s, t)=\sum_{i=1}^{2} a_{3 i}\left(-\frac{\nu_{1} a_{21} \lambda_{1}(s) \cos \vartheta \sinh t}{a_{31}}\right)^{i},
\end{aligned}
$$

with $\nu_{1}=1, \nu_{2}=-1, \lambda_{1}(s)=s^{2}$ and $a_{2 i}=a_{3 i}=1, i=1,2$ such that (3.14) is satisfied. Then we get another member of a surface in $G=S O(3)$ as plotted in Figure 1(b): 


$$
\Psi_{2}(s, t)=\alpha(s)+\sum_{i=1}^{2}\left(t s^{2} \sin \frac{s}{2}\right)^{i} N+\sum_{i=1}^{2}\left(-s^{2} \cos \frac{s}{2} \sinh t\right)^{i} B,
$$

where $\frac{\pi}{6} \leq s \leq \pi, t_{0}=0$ and $-\frac{1}{6} \leq t \leq \frac{1}{6}$.

\section{Conclusions}

In this paper, we study the problem of how to construct surfaces using a line of curvature in a 3-dimensional Lie group. We derive the conditions for the given curve to be the line of curvature for the parametric surface when the marching-scale functions are given by the three types of expressions.

Acknowledgment. The authors thank Editor and the reviewers for their detailed reading of the manuscript and their valuable comments and suggestions that led to a considerable improvement of the paper. The first author was supported by Basic Science Research Program through the National Research Foundation of Korea (NRF) funded by the Ministry of Education (NRF-2018R1D1A1B07046979).

\section{References}

[1] W.J. Che and J.C. Paul, Lines of curvature and umbilical points for implicit surfaces, Comput. Aided Geom. Design, 24 (7), 395-409, 2007.

[2] Ü. Çiftçi, A generalization of Lancret's theorem, J. Geom. Phys. 59 (12), 1597-1603, 2009.

[3] M.P. do Carmo, Differential Geometry of Curves and Surfaces, Englewood Cliffs: Prentice Hall, 1976.

[4] N. do Espírito-Santo, S. Fornari, K. Frensel and J. Ripoll, Constant mean curvature hypersurfaces in a Lie group with a bi-invariant metric, Manuscripta Math. 111 (4), 459-470, 2003.

[5] E. Evren, E. Bayram and E. Kasap, Surface pencil with a common line of curvature in Minkowski 3-space, Acta Math. Sin. (Engl. Ser.) 30 (12), 2103-2118, 2014.

[6] C.Y. Li, R.H. Wang and C.G. Zhu, Parametric representation of a surface pencil with a common line of curvature, Comput. Aided Design, 43 (9), 1110-1117, 2011.

[7] C.Y. Li, R.H. Wang and C.G. Zhu, An approach for designing a developable surface through a given line of curvature, Comput. Aided Design, 45, 621-627, 2013

[8] C.Y. Li, C.G. Zhu and R.H. Wang, A generalization of surface family with common line of curvature, Appl. Math. Comput. 219 (17), 9500-9507, 2013.

[9] C.Y. Li, C.G. Zhu and R.H. Wang, Spacelike developable surfaces through a common line of curvature in Minkowski space, J. Adv. Mech. Des. Syst. Manuf. 9 (4), 1-9, 2015.

[10] T. Maekawa, F.E. Wolter and N.M. Patrikalakis, Umbilics and lines of curvature for shape interrogation, Comput. Aided Geom. Design, 13 (2), 133-161, 1996

[11] T.J. Willmore, An Introduction to Differential Geometry, Oxford University Press, 1959.

[12] D.W. Yoon, General helices of AW(k)-type in the Lie group, J. Appl. Math. 2012, Art. No. 535123, 2012.

[13] X.P. Zhang, W.J. Che and J.C. Paul, Computing lines of curvature for implicit surfaces, Comput. Aided Geom. Design, 26 (9), 923-940, 2009. 